\title{
Eukaryotic microorganisms in microbiology syllabi: better representation needed
}

\section{Opinion}

Through this article, I want to emphasize a point, which is of relevance to all those associated with higher education in the field of Microbiology and associated disciplines. This is about: "The less than required weightage given to eukaryotic microbes in UG and PG Microbiology syllabi"

Though this article is written largely in context of the curriculum followed at Indian universities, is relevant to Microbiology education/ curricula in many other countries.

If one looks at the syllabus of Microbiology courses being taught at UG and/or PG levels in various Indian universities, one can easily find that these syllabi are largely skewed towards bacteriology rather than being a balanced Microbiology curriculum. By latter, I mean a syllabus paying enough attention to microbes other than bacteria, too, i.e. algae, fungi, protozoa, and viruses. This situation partly may be due to the fact that bacteria are among the easiest to handle and grow among all microorganisms, and laboratory teaching using them is somewhat straightforward. For beginners in Microbiology, it is okay to start with these prokaryotic life forms. But then as the course progresses, students must be taught the cultivation and handling of eukaryotic microbes (fungi, algae, and protozoa) too. Though they take more time to grow, a student must be exposed to various aspects of these groups of life forms too.

Currently the laboratory sessions of most UG/PG Microbiology courses teach students to work with bacteria, i.e. how to isolate, cultivate, and identify them, with 1-2 experiments on cultivation and identification of few common fungi. Majority of the Microbiology faculty will be those, who were taught as a student, a Microbiology course largely skewed towards bacteriology, and in turn they can teach only that, and the cycle goes on. Even when any M.Sc. candidate plans for his/her Ph.D., they usually do not think about working with algae or protozoa, because they do not have enough knowhow (and confidence for handling them) about these organisms.

There is an obvious need for a reasonable restructuring of our Microbiology syllabi, which should result in formation of a balanced syllabus with appropriate emphasis (not only theory teaching, but experimental training too) on all members of microbial world (eubacteria, archea, algae, fungi, protozoa, and viruses). As some eukaryotic microorganisms (particularly protozoa) can prove to be good models for testing new drug molecules, paying attention to them can also help in reducing animal sacrifices. Appropriate training programmes for the faculties should also be arranged wherein they can develop the skill for designing experiments involving eukaryotic microorganisms.
Volume I Issue 2 - 2016

\author{
Vijay Kothari \\ Institute of Science, Nirma University, India
}

Correspondence: Vijay Kothari, Institute of Science, Nirma University, India, Email vijay.kothari@nirmauni.ac.in, vijay23112004@yahoo.co.in

Received: September 16, 2016 | Published: October 28, 2016

The process of designing our subject curricula should be responsive to the academic/research needs of the time. For example, earlier fungi were less important as human pathogens; but these days with increase in number of immuno-compromised patients (which is for the reasons like spread of HIV, increasing number of organ transplantations followed by use of immuno-suppressive drugs, increased hospital facilities making the immune-compromised people survive longer, etc.) fungal infections are on a rise. This means that the current and future generations of Microbiology students need to be offered a syllabus which emphasizes on fungi and their pathogenicity, more than the older day syllabus. Another such example is that till recently the biofilm mode of microbial life was not given that high attention by the community of microbiologists, but now it is widely accepted that in nature (and inside their human/non-human hosts, too) microorganisms do prominently live a biofilm-lifestyle, wherein their gene-expression is quite different than their free-living counterparts. Thus it is logically required for any modern microbiology course to give enough emphasis (theoretical as well as experimental) on microbial biofilms. These are just two examples, but the syllabus designing bodies (i.e. the Board of Studies) at universities need to be responsive to such changes in human lifestyle and knowledge level, which make certain aspects of Microbiology more relevant than earlier; so that our students can be offered more meaningful curricula.

\section{Acknowledgements}

None.

\section{Conflict of interest}

The author declares no conflict of interest. 